\title{
Correction: U.S. adults are not meeting recommended levels for fish and omega-3 fatty acid intake: results of an analysis using observational data from NHANES 2003-2008
}

Yanni Papanikolaou ${ }^{1 *}$, James Brooks ${ }^{2}$, Carroll Reider ${ }^{3}$ and Victor L Fulgoni ${ }^{4}$

\section{Correction}

Following the publication of this article [1], we noted errors to Tables five and seven (Tables 1 and 2 here). Corrected versions are presented below.

Table 1 Mean usual intake of EPA from foods ( $\mathrm{mg} / \mathrm{day}$ ) in adults from NHANES 2003-2008

\begin{tabular}{|c|c|c|c|c|c|c|c|c|c|}
\hline \multirow[b]{2}{*}{ Gender } & \multirow[b]{2}{*}{ Age } & \multirow[b]{2}{*}{$\mathrm{N}$} & \multicolumn{2}{|c|}{ Usual Intake } & \multicolumn{5}{|c|}{ Percentile } \\
\hline & & & Mean & SE & 10 & 25 & 50 & 75 & 90 \\
\hline All & $19+$ Years & 14,338 & 23 & 1 & 7 & 11 & 18 & 29 & 43 \\
\hline All & $19-50+$ Years & 7,585 & 23 & 1 & 7 & 11 & 18 & 29 & 44 \\
\hline All & $51+$ Years & 6,753 & 22 & 1 & 8 & 12 & 18 & 28 & 42 \\
\hline Male & $19+$ Years & 7,302 & 27 & 1 & 9 & 14 & 22 & 34 & 51 \\
\hline Male & $19-50+$ Years & 3,944 & 28 & 2 & 9 & 14 & 23 & 35 & 52 \\
\hline Male & $51+$ Years & 3,358 & 26 & 2 & 9 & 13 & 21 & 33 & 49 \\
\hline Female & $19+$ Years & 7,036 & 18 & 1 & 7 & 10 & 15 & 23 & 34 \\
\hline Female & $19-50+$ Years & 3,641 & 18 & 1 & 6 & 9 & 15 & 23 & 33 \\
\hline Female & $51+$ Years & 3,395 & 19 & 1 & 7 & 11 & 16 & 25 & 36 \\
\hline
\end{tabular}

Table 2 Mean usual intake of DHA from foods (mg/day) in adults from NHANES 2003-2008

\begin{tabular}{|c|c|c|c|c|c|c|c|c|c|}
\hline \multirow[b]{2}{*}{ Gender } & \multirow[b]{2}{*}{ Age } & \multirow[b]{2}{*}{$\mathrm{N}$} & \multicolumn{2}{|c|}{ Usual Intake } & \multicolumn{5}{|c|}{ Percentile } \\
\hline & & & Mean & SE & 10 & 25 & 50 & 75 & 90 \\
\hline All & $19+$ Years & 14,338 & 63 & 2 & 21 & 32 & 50 & 79 & 119 \\
\hline All & $19-50+$ Years & 7,585 & 63 & 2 & 20 & 31 & 50 & 80 & 120 \\
\hline All & $51+$ Years & 6,753 & 62 & 2 & 21 & 32 & 50 & 78 & 116 \\
\hline Male & $19+$ Years & 7,302 & 75 & 3 & 25 & 39 & 61 & 95 & 140 \\
\hline Male & $19-50+$ Years & 3,944 & 77 & 3 & 26 & 40 & 63 & 98 & 145 \\
\hline Male & $51+$ Years & 3,358 & 71 & 4 & 24 & 37 & 58 & 90 & 132 \\
\hline Female & $19+$ Years & 7,036 & 51 & 2 & 18 & 27 & 42 & 64 & 94 \\
\hline Female & $19-50+$ Years & 3,641 & 48 & 2 & 17 & 26 & 40 & 61 & 89 \\
\hline Female & $51+$ Years & 3,395 & 54 & 3 & 19 & 29 & 45 & 68 & 100 \\
\hline
\end{tabular}

* Correspondence: papanikolaou.yanni@gmail.com

'Nutritional Strategies Inc, 59 Marriot Place, Paris, ON N3LOA3, Canada
We also noted that the third paragraph of the Discussion states that our data shows daily consumption of EPA and DHA of $0.41 \mathrm{~g}$ and $0.72 \mathrm{~g}$, respectively, however this should be $41 \mathrm{mg} /$ day and $72 \mathrm{mg} /$ day, respectively.

Lastly, the titles of Tables six and eight in the published manuscript should be displayed as, "Mean usual intake of EPA from foods and dietary supplements (mg/day) in adults from NHANES 2003-2008" and "Mean usual intake of DHA from foods and dietary supplements ( $\mathrm{mg} /$ day) in adults from NHANES 2003-2008", respectively.

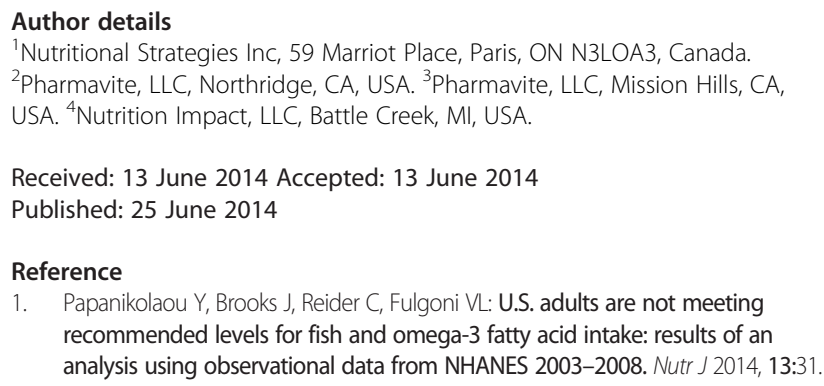

1. Papanikolaou Y, Brooks J, Reider C, Fulgoni VL: U.S. adults are not meeting recommended levels for fish and omega-3 fatty acid intake: results of an analysis using observational data from NHANES 2003-2008. Nutr J 2014, 13:31.

\section{doi:10.1186/1475-2891-13-64}

Cite this article as: Papanikolaou et al:: Correction: U.S. adults are not meeting recommended levels for fish and omega-3 fatty acid intake: results of an analysis using observational data from NHANES 2003-2008. Nutrition Journal 2014 13:64. 